\title{
Speech disorders in children: Our experience in a tertiary care teaching hospital in eastern India
}

\author{
Santosh Kumar Swain 1 , Mahesh Chandra Sahu², Jasashree Choudhury ${ }^{3}$ \\ 'Department of Otorhinolaryngology, IMS and SUM Hospital, Siksha "O" Anusandhan University, K8, Kalinganagar, \\ Bhubaneswar, Odisha, India \\ 2Directorate of Medical Research, IMS and SUM Hospital, Siksha "O" Anusandhan University, K8, Kalinganagar, Bhubaneswar, \\ Odisha, India \\ ${ }^{3}$ Department of Paediatrics, IMS and SUM Hospital, Siksha "O" Anusandhan University, K8, Kalinganagar, Bhubaneswar, \\ Odisha, India
}

\section{ABSTRACT}

Introduction: A wide variety of speech disorders exist, which impair the ability to communicate among children and adversely affect the social, emotional, and functional well-being of the child.

Aim of the study: To find out speech disorders in routine paediatric otorhinolaryngology practice and the role of speech therapy for its effective management.

Material and methods: This study was carried out on 58 children who presented with speech disorders at the outpatient and indoor of the department of otorhinolaryngology.

Results: In this study, speech disorders like stuttering, dysglossia, dyslalia, deaf-mutism, and mouth breathing were prevalent in both sexes but encountered more in males compared to females. Stuttering was the most commonly encountered speech disorder in which speech therapy provided encouraging results. All dyslalia children underwent appropriate articulation training and improved satisfactorily, except one. All deaf-mute children $(n=9)$ underwent auditory training and speech and language stimulation therapy, following which four patients acquired satisfactory speech levels. All dysglossia or tongue-tie children improved satisfactorily after tongue-tie release surgery with appropriate speech therapy.

Conclusions: Newer treatment modalities and coordination between otorhinolaryngologists and speech pathologists are helping to achieve correct diagnosis and treatment of speech disorders. Speech therapy is often suitably individualised according to the patient's needs. A detailed understanding of the effectiveness of speech therapy has emerged as an acceptable modality of treatment in different speech disorders.

KEY WORDS:

speech disorder, speech therapy, rehabilitation.

\section{INTRODUCTION}

Early childhood is an important time in life for the development of speech. Speech is the vocalised form of language of human beings, which makes him/her a social animal. Speech is the articulated form of sounds or words used to convey meanings. Speech is referred to as having a disorder or being defective when it differs significantly outside the range of acceptable changes in the same community, of the same age group and sex, with underlying conditions making such a deviation [1]. There are variety of speech disorders that impair the ability to communicate to various extents and adversely affect the emotional, social, and functional well-being of a child. Children with

\section{ADDRESS FOR CORRESPONDENCE:}

Santosh Kumar Swain, Department of Otorhinolaryngology, IMS and SUM Hospital, Siksha "O” Anusandhan

University, K8, Kalinganagar, Bhubaneswar, Odisha, India, e-mail: santoshvoltaire@yahoo.co.in 
speech disorders often need a multidisciplinary approach in a Paediatric Otorhinolaryngology Department because hearing examination, oral cavity (tongue) examination, laryngeal examination, and mental status are important for proper assessment of speech. In children with normal hearing, a cognitive delay may cause a language delay [2]. The option of speech therapy in children is often based on the wishes of the parents or guardians because they usually believe that speech therapy is the best option for their child. We carried out a study on speech disorders encountered in a paediatric group, and on the role of speech therapy for its effective management.

\section{MATERIAL AND METHODS}

Fifty-eight children presented to the Department of Otorhinolaryngology for speech disorders. All the children were evaluated by the Speech and Audiology Unit of the Otorhinolaryngology Department. The period of the study was from July 2015 to August 2017. Detailed history and complete ENT examination were done and documented. The details of developmental milestones and clinical history were obtained from the parents of the children. The speech behaviour of all the children, hearing assessment, any articulation defect, vocabulary mode of communication, and disfluencies were documented. The writing, reading, and comprehensive abilities were examined for assessment of language. Functions and appearance of peripheral organs of speech including diadochokinesis of tongue and lips were noted. The children who were suffering from stammering read a standard text of local language, and the occurrence of manifestations of stammering were documented. After complete reading of the text, every child was asked to narrate what they had read, and the responses were again noted. Children were asked to recite any story they had learnt, and their spoken words were observed. Children were also asked about the situations when they stammered most, e.g. with friends, strangers, or family members. Stammerers were trained with different breathing exercises. Delayed speech and language developments without any aetiology need speech therapy and different occupational and physical therapies. Parents or guardians of such children were instructed to train them by reading text regularly in loud and clear voice. Positive reinforcement in the form of appreciation and reward of/for the child are part of the therapy. In our study, all the children were instructed for follow-up and speech therapy sessions every week.

\section{RESULTS}

Speech disorders are prevalent in both sexes, although they occur more in males than in females. This study included a total of 58 children: 39 boys and 19 girls, in a ratio of 2.05: 1 (Table 1). Speech disorders like stuttering, dyslalia, deaf-mutism, and dysglossia were common in our study. Stuttering was the most commonly encountered speech disorder where speech therapy had encouraging results. All dyslalia children underwent appropriate articulation training and improved satisfactorily, except one child. All deaf-mute children underwent auditory training and speech and language stimulation therapy, following which four children acquired satisfactory speech levels. All dysglossia or tongue-tie children improved satisfactorily after tongue-tie release surgery with appropriate speech therapy.

\section{DISCUSSION}

Speech disorders are often a hidden disability affecting the social and emotional well-being, cognition, behaviour, and academic excellence in the school of the child. An untreated speech disorder during childhood may affect vocational choices in adulthood [3]. Speech disorders in children may be congenital or acquired, and functional or organic in origin. Some may result from purely psychological causes, and some may result from surgery on speech organs. When the speech disorder occurs due to hearing impairment, the problem becomes more serious in nature and may need extensive treatment.

The communication disorders in children are grossly divided into five categories: developmental speech and language disorders, speech disorders, language disorders, disorders of fluency, and disorders of voice. Major devel-

TABLE 1. Different speech disorders seen among children in our study

\begin{tabular}{|l|c|c|c|}
\hline Speech disorder & Male (\%) & Female (\%) & Total \\
\hline Stuttering & $10(71.42)$ & $4(28.57)$ & 9 \\
\hline Dyslalia & $7(77.77)$ & $2(22.22)$ & 9 \\
\hline Deaf-mute & $6(66.66)$ & $3(33.33)$ & 8 \\
\hline Tongue-tie & $5(62.5)$ & $3(37.5)$ & 7 \\
\hline Delayed speech and language (idiopathic) & $4(57.14)$ & $3(42.85)$ & 5 \\
\hline Mental retardation & $3(60)$ & $2(405)$ & 6 \\
\hline Mouth breathers & $4(66.66)$ & $2(33.33)$ & \\
\hline
\end{tabular}


opmental speech and language disorders include: idiopathic delayed speech and language (DSL), cerebral palsy $(\mathrm{CP})$, mental retardation (MR), and hearing impairment (HI). Hearing impairment disorders constitute a large proportion of developmental disorders [4]. Speech disorders can have different types, and the presentations may affect both speech and language. Disturbed fluency (stuttering), impaired production of speech (misarticulation), and voice disorders are a few that affect communication in daily life. The speech disorders also include language impairment like aphasia and certain speech problems, which lead to an inability to follow the content, form, and function of the language [5]. These disorders may occur in any age group, but the speech disorders are often seen in childhood, affecting 1 in every 12 children or $5-8 \%$ of pre-school children [6].

It was seen that the highest number of speech disorders were stuttering. There were certain factors associated with children with stuttering, and these were: emotionally labile and sensitive nature, family attitude, self-confidence, peer pressure, family atmosphere, and awareness among the parents. All the stuttering children were treated with speech therapy (progressive muscular relaxation therapy, modified mean airflow technique). Parents and guardians of stuttering children were often educated for awareness of stuttering and their role in the treatment of stuttering. Speech disorders among children are often assessed by parents, school teachers, and family doctors. They can rate the child's speech as "fully intelligible", "almost all words intelligible", "many words unintelligible", or "almost all words unintelligible". Children are often assessed for stammering by asking family members. The school teacher reported about school situations in which there were any difficulties in understanding the child due to poor speech, and if so, whether the speech of the child was "somewhat" or "certainly" difficult to understand. Out of 14 stuttering children, 11 patients improved and three patients did not improve satisfactorily. Out of the three children who did not improve, two of them did not perform their speech therapy practice regularly at home and were not supported well by the family. This suggests that a multidisciplinary approach from speech therapists, otolaryngologists, and family members is necessary for a favourable outcome for stuttering treatment. Out of five children with mental retardation, all were treated by speech therapy and behavioural training. Three children with mental retardation improved, while two children were lost to follow-up. Speech therapy along with special education gives a better outcome in speech disorders with mental retardation.

Rehabilitation of deaf-mute children always poses a challenge to speech pathologists. All deaf-mute children in our study were given auditory training and speech and language stimulation therapy. Mouth breathing in children is also an aetiology for speech disorders. Due to chronic mouth breathing, there are alterations in cranio-facial growth, speech, body posture, sleep quality, and school performance [7]. Overall, the mouth breather shows alterations in posture, mobility, and tone of lips, tongue, and cheek, leading to less efficiency of speech, flaccid jaw elevator muscles, and speech disorders [8].

Speech is altered due to flaccid facial muscles, defective positioning of the tongue, or structural defects in the oral cavity due to malocclusion and deficiencies in facial growth and development [9]. The most common speech disorders in mouth breathers are anterior positioning tongue during production of lingual dental phonemes and imprecision in labial and fricative phonemes [10].

In our study, all the tongue-tie children (dysglossia) causing articulation disorders showed defects of linguadental and alveolar sounds as well as sibilants like 'd, ' $t$ ', 'l, 's', 'z', etc. In dyslalia patients, it was seen that the substituted sound is often one of the sounds acquired earlier during the sequence of speech development, e.g. ' $w$ ' for ' $r$ ' as in 'wan' for 'ran', ' $t$ ' for ' $k$ ' as in 'titty' for 'kitty'. It was also seen that diadochokinetically faster sounds ' $t$ ', ' $d$,' ' $p$ ', and 'b' were substituted for slower sounds [11]. All of our dyslalia children were subjected to articulation training, following which all patients were improved where one patient missed follow-up.

Out of seven children with delayed speech and language development, three were preterm babies and two had neonatal jaundice. It was seen that delayed physical milestones and less intelligence quotient were also associated with delayed speech and language development [12]. All of the children with delayed speech and language were treated with speech therapy, and out of seven, five children improved and two did not attend follow-up regularly.

There is a new insight in which language plays an important role in social-emotional and cognitive development. Early treatment of speech and language disorders is thought to prevent speech and learning problems, dyslexia, and behavioural problems [13]. Speech therapy plays a key role in young children with speech disorders and should be started as early as possible. Future research is needed for speech problems in children with more emphasis on treatment.

\section{CONCLUSIONS}

Newer treatment options and good coordination among otorhinolaryngologists and speech-pathologists can help in the correct diagnosis of speech disorders and their treatment. Speech therapy is individualised according to the expectations of the patient and the limitations of the speech therapists. The effectiveness of speech therapy in speech disorders depends on the cooperation of the child, expertise of speech therapist, motivation and patience of the family members of the patient, and very good coordination between speech pathologists and otorhinolaryngologists. Thus, adequate pre-speech therapy 
work-up of the children, realistic expectations among family members, and effective speech therapy result in better outcomes.

\section{DISCLOSURE}

The authors declare no conflict of interest.

\section{REFERENCES}

1. Dave J, Sinha V, Barot D, et al. Speech disorders encountered in routine ENT practice and the role of speech therapy in its effective management. Indian J Otology 2013; 19: 169-172.

2. Siena M, Brouwer G, Knijff WA. Efficacy of speech therapy in children with language disorders: specific language impairment compared with language impairment in comorbidity with cognitive delay. Int J of Pediatric Otorhinolaryngol 2002; 63: 129-136.

3. McKinnon DH, McLeod S, Reilly S. The prevalence of stuttering, voice and speech-sound disorders in primary school students in Australia. Lang Speech Hear Serv Sch 2007; 38: 5-15.

4. Dey R, Kumar S, Kumar T, Davessar JL. Variety of speech and language disorders reportingat a tertiary care hospital in Malwa belt of Punjab, India. Clin Epidemiol Glob Health 2017; 5: 48-51.

5. Definitions of communication disorders and variations. AdHoc Committee on Service Delivery in the Schools. American Speech-Language-Hearing Association. ASHA Suppl 1993; 35 (3 Suppl. 10): 40-41.

6. US Preventive Services Task Force. Screening for speech and language delay in preschool children: recommendation statement. Pediatrics 2006; 117: 497-501.

7. Branco A, Ferrari GF, Weber SA. Orofacial alterations in allergic diseases of the airways. Rev Paul Pediatr 2007; 25: 266-270

8. Hitos SF, Arakaki R, Solé D, Weckx LL. Oral breathing and speech disorders in children. J Pediatr (Rio J) 2013; 89: 361-365.

9. Martinelli RL, Fornaro EF, Oliveira CJ, et al. Correlations between speech disorders, mouth breathing, dentition and occlusion. Rev CEFAC 2011; 13: 17-26.

10. Monteiro VR, Brescovici SM, Delgado SE. The occurrence of lisp in eight- to 11-year-old children from municipal schools. Rev Soc Bras Fonoaudiol 2009; 14: 213-218.

11. Schneider SL, Sataloff RT. Voice therapy for the professional voice. Otolaryngol Clin North Am 2007; 40: 1133-1149.

12. Morgan CT. Introduction to psychology.7th ed. McGraw Hill; New York 2010: 531-532.

13. Coster FW. Behavioural Problems in Children with Specific Language Impairments, Stichting Kinderstudies, Groningen 2001. 